[Chem. Pharm. Bull.

33( 7 )2654-2662(1985)

\title{
Distribution of Acetylcholine Chloride between the Micellar and Bulk Phases as Studied by Ultrafiltration ${ }^{1)}$
}

\author{
MASAYUKi NAKagaKi and SHOKo YokoYama* \\ Faculty of Pharmaceutical Sciences, Kyoto University, Yoshida- \\ Shimoadachi-cho, Sakyo-ku, Kyoto 606, Japan
}

(Received October 25, 1984)

\begin{abstract}
The distribution of acetylcholine chloride (Ach) between the aqueous and micellar phases has been measured by means of ultrafiltration and the binding parameters have been estimated. The results indicate that Ach enters not only anionic micelles of sodium dodecyl sulfate (SDS), but also cationic micelles of dodecyltrimethylammonium chloride (DTAC). Furthermore, in the cases of ionic micelles and mixed micelles composed of ionic and nonionic, (heptaethylene glycol dodecyl ether (HED)), surfactants, it has been found that Ach molecules are bound to one locus within the surfactant micelles. The Ach molecule has higher affinity but lower saturated adsorption amount for DTAC micelles than SDS micelles, indicating that Ach molecules are probably situated at the surface of the SDS micelles, but penetrate more deeply and bind more tightly to DTAC micelles. For nonionic micelles, the bound Ach concentration (percent) was very small, but for mixed micelles, the percentage increased with mole fraction of ionic surfactants. Furthermore, in the system of negatively charged mixed micelles and Ach, the bound Ach concentration (percent) was found to depend on the surface potential of the micelles, and the dependence was explained well by the Gouy-Chapman theory.
\end{abstract}

Keywords-_-ultrafiltration; distribution; acetylcholine chloride; sodium dodecyl sulfate; dodecyltrimethylammonium chloride; micelle; mixed micelle; surface potential; surface charge density; Gouy-Chapman theory

It is important in various fields to know how much of a substance is partitioned into the micellar phase. For this purpose, studies have been carried out by means of the solubility method, ${ }^{2)}$ fluorescent probe method, ${ }^{3)}$ calorimetric method, ${ }^{4)}$ gas chromatographic technique $^{5)}$ and dialysis method ${ }^{6)}$ Recently, the ultrafiltration method, which is direct and simple, has been widely applied, for example, to monomer-micellar equilibrium of aqueous surfactant (sodium alkyl benzene sulfonates) solutions, ${ }^{7}$ the distribution of methyl orthobenzoate, 1fluoro-2,4-dinitrobenzene and aniline between water and micelles of sodium dodecyl sulfate and a dinonylphenyl polyoxyethylene surfactant ${ }^{8)}$ and the distribution of $p$-hydroxybenzoate, which is a preservative, between the aqueous and micellar phases. ${ }^{9)}$

These distributions are affected by both the hydrophobic character of substances and the electrostatic interaction between ionic surfactants and ionized substances. Therefore, the penetration of an ionized substance into an ionic surfactant micelle of the same sign by hydrophobic interaction may be especially interesting. Furthermore, it is of interest to study the reaction at the micelle surface as a model of chemical reactions at the surface of biological membranes. We have studied the hydrolysis of acetylcholine chloride, which is a well-known chemical messenger and drug, in the presence of anionic, cationic and nonionic surfactants. ${ }^{10)}$ For accurate analysis of the result, it is absolutely necessary to know the distribution of acetylcholine chloride between the aqueous and micellar phases.

From these points of view, we studied the distribution of acetylcholine chloride in anionic and cationic micelles and estimated the binding parameters. The results are presented here. 


\section{Experimental}

Materials-Sodium dodecyl sulfate (SDS) supplied by Nihon Emulsion Co. and dodecyltrimethylammonium chloride (DTAC) purchased from Nakarai Chemicals Co. were purified as described previously. ${ }^{11)}$ Heptaethylene glycol dodecyl ether (HED) purchased from Nikkol Chemicals was of guaranteed reagent grade and was used without further purification. Acetylcholine chloride (Ach), hydroxylamine hydrochloride and ferric chloride $\left(\mathrm{FeCl}_{3}\right)$ purchased from Nakarai Chemicals Co. were of guaranteed reagent grade and were used without further purification. The Diafio membrane, YC-05, $43 \mathrm{~mm}$ i.d., a cellulose acetate membrane, was purchased from Amicon Corp., Mass., U.S.A.

Ultrafiltration - The technique employed was essentially the same as that described by Shimamoto et al. ${ }^{9 a}$ This is based on the fact that Ach bound to micelles is not filtered through the YC- 05 membrane. We confirmed by the dye method using pinacyanol chloride that micelles could not pass through the filter. The ultrafiltration cell was filled with $10 \mathrm{ml}$ of the test solution. The effluent was collected in 0.1 or $0.2 \mathrm{ml}$ portions. All procedures were carried out at room temperature.

Aqueous solutions of Ach were filtered through the membrane, and the Ach concentration of the filtrate reached the same level as the initial concentration after $0.5 \mathrm{ml}$. It can, therefore, be assumed that the membrane is satisfactorily nonretentive for Ach. The time lag may be a consequence of the "void volume" of the system as pointed out by Blatt et al. ${ }^{121}$

In this study, the volume of filtrate is small compared to the whole volume, and the time spent during ultrafiltration is short. Furthermore, no deterioration of the YC-membrane by the surfactants was found, although Shimamoto et al. ${ }^{9 a)}$ reported deterioration of the UM-membrane (a cross-linked dextran gel membrane, Amicon Corp., Mass., U.S.A.). Therefore, ultrafiltration through the YC-membrane is considered to be an adequate method for the measurement of the binding characteristics of Ach to surfactants.

Quantitative Analysis of Ach_-After ultrafiltration, the concentration of Ach was determined spectrophotometrically at a wavelength of $520 \mathrm{~nm}$ as described by Hestrin, ${ }^{13)}$ namely,

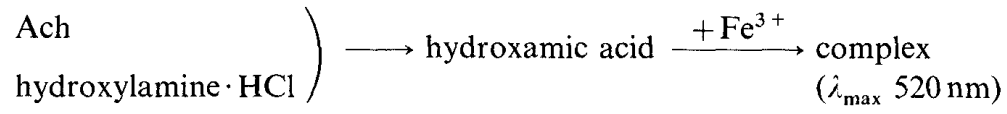

The relation between concentration and absorbance was found to obey Beer's law at least up to $8 \mathrm{mmol} \cdot \mathrm{dm}^{-3} \mathrm{of}$ Ach, and the molar absorptivity $\left(\varepsilon_{520}\right)$ was obtained as 208 .

The effect of interfering substances capable of binding to ferric ion (ex. sulfate), in the quantitative analysis, was overcome by the use of a sufficient concentration of $\mathrm{FeCl}_{3}, 0.37 \mathrm{~mol} \cdot \mathrm{dm}^{-3}$.

\section{Results}

\section{Free Ach Concentration (Percent) in the Presence of Ionic Surfactants}

The concentration of free Ach, $\left[D_{\mathrm{f}}\right]$, was determined as the Ach concentration in the ultrafiltrate. The percentages of free Ach concentration with respect to the total con-

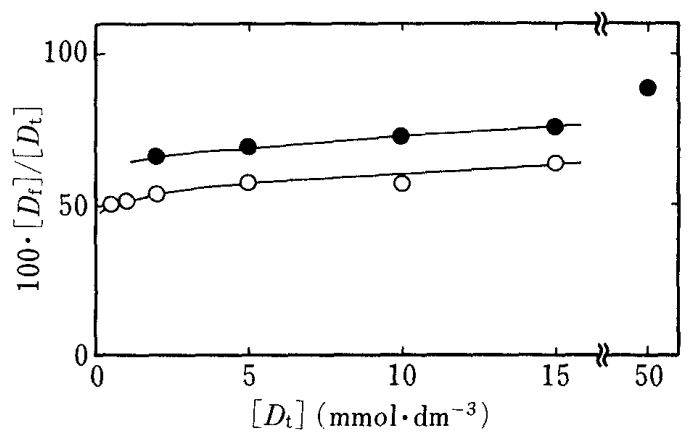

Fig. 1. Changes in Free Ach Concentration (Percent) in the Aqueous Phase, $\left[D_{\mathrm{f}}\right]$, at Various Total Concentrations of Ach, $\left[D_{\mathrm{t}}\right]$, in SDS Solutions

$$
\text { Concentration of } \operatorname{SDS}\left(C_{\mathrm{SDS}}\right):-20 ; 0,30
$$
$\mathrm{mmol} \cdot \mathrm{dm}^{-3}$.

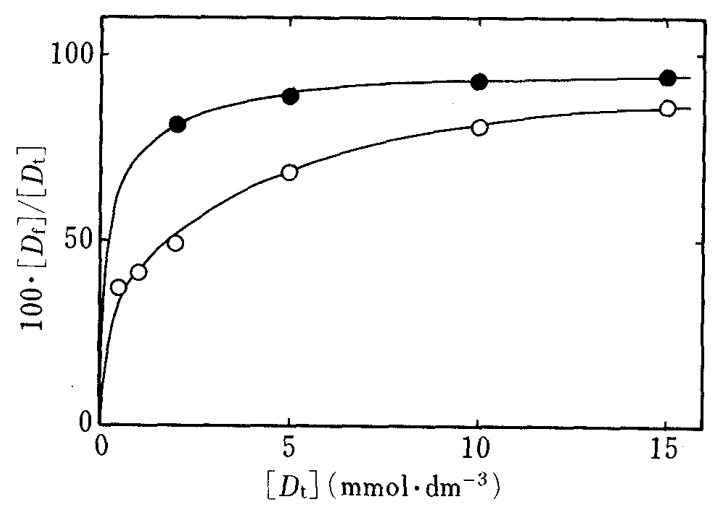

Fig. 2. Changes in Free Ach Concentration (Percent) at Various Total Concentrations of Ach in DTAC Solutions

Concentration of DTAC $\left(C_{\text {DTAC }}\right):-20 ; 0,30$ $\mathrm{mmol} \cdot \mathrm{dm}^{-3}$. 

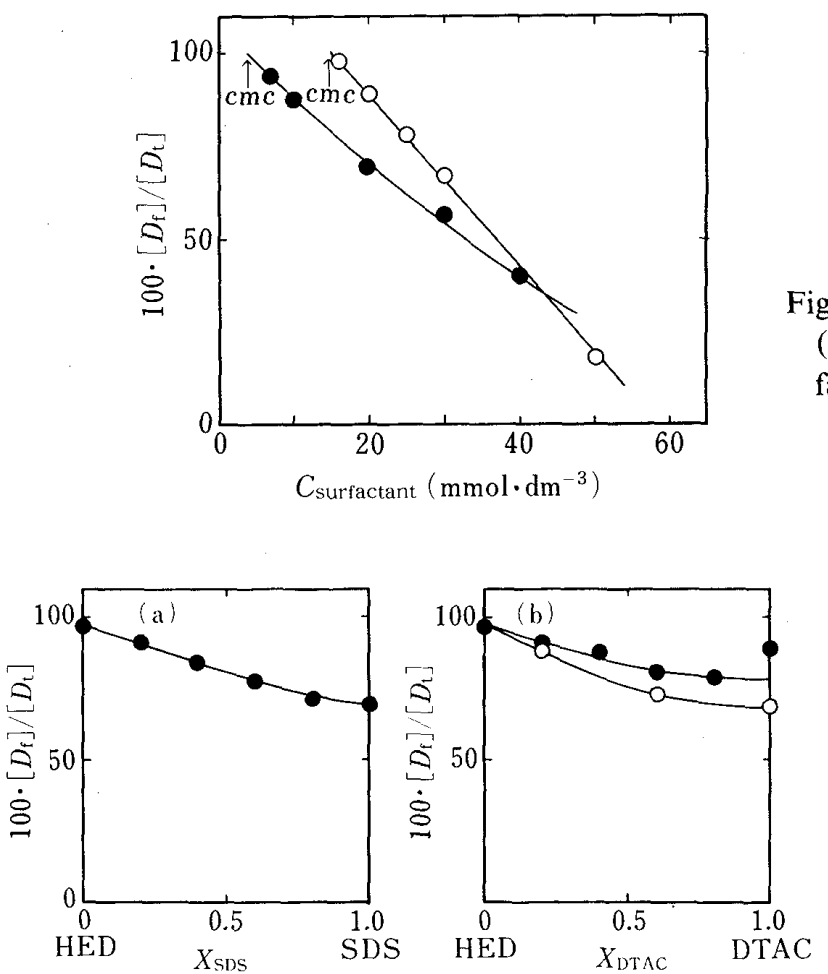

Fig. 4. Changes in Free Ach Concentration (Percent) at Various Mole Fractions of Ionic Surfactant

(a) HED-SDS system; (b) HED-DTAC system. Total conc. of surfactants:, $20 ; \bigcirc, 30 \mathrm{mmol}$ $\mathrm{dm}^{-3} \cdot\left[D_{\mathrm{t}}\right]: 5 \mathrm{mmol} \cdot \mathrm{dm}^{-3}$.
Fig. 3. Changes in Free Ach Concentration (Percent) at Various Concentrations of Surfactants

Surfactants: $O$, DTAC; $\boldsymbol{\bullet}$, SDS.

$\left[D_{\mathrm{t}}\right]: 5 \mathrm{mmol} \cdot \mathrm{dm}^{-3}$

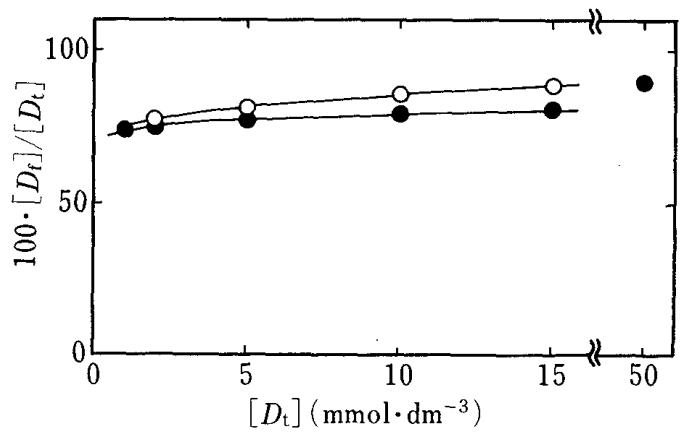

Fig. 5. Changes in Free Ach Concentration (Percent) at Various Total Concentrations of Ach in Mixed Surfactant Systems

Mixed surfactant system: $\bigcirc X_{\mathrm{DTAC}}=0.6, \odot X_{\mathrm{SDS}}=$
0.6.
Total surfactant concentration: $20 \mathrm{mmol} \cdot \mathrm{dm}^{-3}$.

centration, $100\left[D_{\mathrm{f}}\right] /\left[D_{\mathrm{t}}\right]$, in the presence of SDS and DTAC are shown in Figs. 1 and 2, respectively. Ach is distributed not only in the SDS micelles but also in the DTAC micelles, in spite of the electrostatic repulsion between the head groups of Ach cations and DTA cations. The percentage of free Ach concentration increases with the total concentration of Ach in both cases, though the increase is small in the presence of SDS, but large in the presence of DTAC. The decrease of the percentage of free Ach concentration at various concentrations of SDS and DTAC at a constant Ach concentration of $5 \mathrm{mmol} \cdot \mathrm{dm}^{-3}$ is illustrated in Fig. 3. The critical micelle concentrations ( $\mathrm{cmc}$ ) of SDS and DTAC, at this Ach concentration, indicated in Fig. 3, have been reported previously. ${ }^{11 c)}$

\section{Free Ach Concentration (Percent) in the Presence of Mixed Micelle Systems}

The percentage of free Ach concentration with respect to total concentration, $100\left[D_{\mathrm{f}}\right] /$ $\left[D_{t}\right]$, in the presence of mixed micelles is illustrated in Fig. 4, as a function of either $X_{\mathrm{SDS}}$ or $X_{\text {DTAC }}$, the mole fraction of ionic surfactant in the mixtures with the nonionic surfactant, HED. In the case of nonionic surfactant alone, Ach molecules exist almost entirely in the free form, although the value of $100\left[D_{\mathrm{f}}\right] /\left[D_{\mathrm{t}}\right]$ decreases with increase in $X_{\mathrm{SDS}}$ or $X_{\mathrm{DTAC}}$. The very small binding to the nonionic micelles at zero $X_{\mathrm{SDS}}$ or $X_{\mathrm{DTAC}}$ may be due to the polyoxyethylene mantle, which is less hydrophobic and hinders the hydrophobic interaction of Ach with the nonionic micelles. The percentage value obtained at a concentration of $20 \mathrm{mmol} \cdot \mathrm{dm}^{-3}$ of DTAC (at $X_{\text {DTAC }}=1$ ) is not on the extrapolated smooth curve. This is probably because the amount of DTAC micelles was not sufficient, since the cmc of DTAC in the presence of Ach of $5 \mathrm{mmol} \cdot \mathrm{dm}^{-3}$ is approximately $15 \mathrm{mmol} \cdot \mathrm{dm}^{-3} \cdot{ }^{11 c)}$ For $30 \mathrm{mmol}$. $\mathrm{dm}^{-3}$ total surfactant concentration, all plots are on the curve. 
At a mole fraction of 0.6 of ionic surfactant, the percentage of free Ach concentration is shown in Fig. 5. The percentage of free Ach concentration slightly increases with the concentration of Ach in both cases, but the degree of increase is smaller than that in the solutions of ionic surfactants alone (Figs. 1 and 2).

\section{Adsorption Amount and Binding Constant of Ach for Ionic Micelles}

If the Langmuir adsorption isotherm is assumed to be applicable to the adsorption of Ach by surfactant micelles, the equation may be written as follows:

$$
x=\frac{\left[D_{\mathrm{b}}\right]}{[S]}=\frac{x_{\infty} k\left[D_{\mathrm{f}}\right]}{1+k\left[D_{\mathrm{f}}\right]}
$$

where $x$ is the adsorption amount of Ach per mole of surfactant in micelles, $x_{\infty}$ is the saturated amount of adsorption, $k$ is the association constant for the adsorption, $[S]$ is the concentration of surfactant which is in the form of micelles and $\left[D_{\mathrm{b}}\right]$ is the concentration of bound Ach. Then, $\left[D_{\mathrm{b}}\right]=\left[D_{\mathrm{t}}\right]-\left[D_{\mathrm{f}}\right]$, where $\left[D_{\mathrm{f}}\right]$ is the concentration of free Ach, as determined by ultrafiltration. The concentration of micellar surfactant $[S]$ can be obtained accurately by using the values of critical micelle concentration of the surfactant in the presence of various concentrations of Ach. ${ }^{11 c)}$

The adsorption amounts, $x$, obtained from Figs. 1 and 2 are plotted against the values of $\left[D_{\mathrm{f}}\right]$ in Fig. 6(a) for SDS and in 7(a) for DTAC. The values of $x$ fall on the same curve irrespective of the surfactant concentration and increase with the concentration of free Ach.

Equation 1 can be transformed as follows:

$$
\frac{x}{\left[D_{\mathrm{f}}\right]}=k\left(x_{\infty}-x\right)
$$

The plots of $x /\left[D_{\mathrm{f}}\right]$ against $x$ for SDS and DTA are shown in Figs. 6(b) and 7(b), respectively. These Scatchard plots give good linear relationships, indicating that Ach molecules are bound to one loci within the micelles of SDS or DTAC. Furthermore, $k$ and $x_{\infty}$ can be obtained from the slope and intercept (footnote $a$ in Table I).

\section{$\boldsymbol{k}$ and $\boldsymbol{x}_{\infty}$ of Ach for the Mixed Micelles}

The binding parameters of Ach for the negatively charged and positively charged mixed

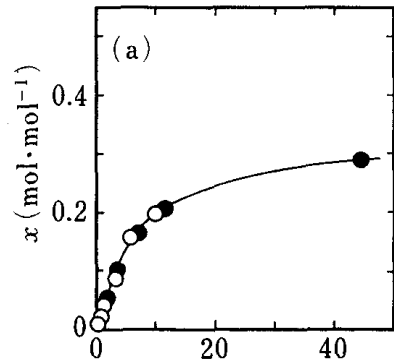

$\left[D_{\mathrm{f}}\right]\left(\mathrm{mmol} \cdot \mathrm{dm}^{-3}\right)$

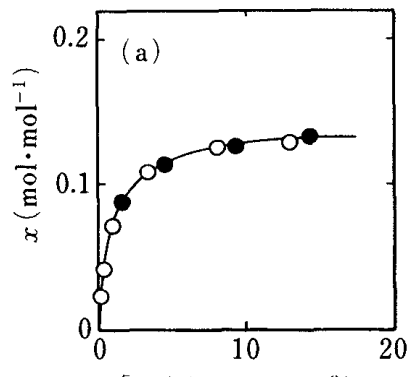

$\left[D_{\mathrm{f}}\right\rfloor\left(\mathrm{mmol} \cdot \mathrm{dm}^{-3}\right)$

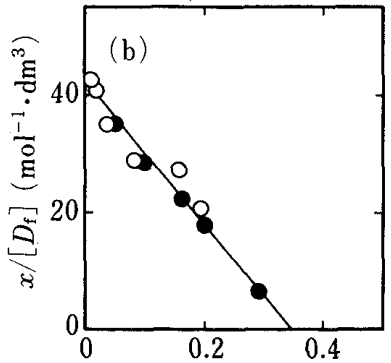

$x\left(\mathrm{~mol} \cdot \mathrm{mol}^{-1}\right)$

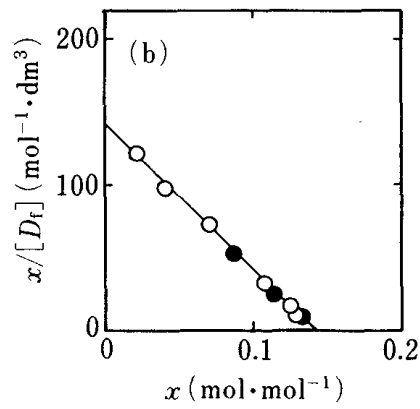

Fig. 6. Relation between Bound Amount, $x$, and Concentration of Free Ach (a), and the Scatchard Plot (b) for the Interaction of Ach with SDS

$C_{\mathrm{SDS}}:-20 ; \bigcirc, 30 \mathrm{mmol} \cdot \mathrm{dm}^{-3}$.

Fig. 7. Relation between $x$ and $\left[D_{\mathrm{t}}\right](\mathrm{a})$, and the Scatchard Plot (b) for the Interaction of Ach with DTAC

$C_{\text {DTAC }}: 20 ; 0,30 \mathrm{mmol} \cdot \mathrm{dm}^{-3}$. 
TABLE I. Values of $k$ and $x_{\infty}$ of Ach for Surfactant Micelles

\begin{tabular}{|c|c|c|}
\hline Surfactant micelles & $k\left(\mathrm{~mol}^{-1} \cdot \mathrm{dm}^{3}\right)$ & $x_{\infty}\left(\mathrm{mol} \cdot \mathrm{mol}^{-1}\right)$ \\
\hline SDS & $120^{a)}$ & $0.34^{a)}$ \\
\hline HED-SDS $\left(X_{\mathrm{SDS}}=0.6\right)$ & 48 & 0.37 \\
\hline DTAC & 944 & 0.14 \\
\hline HED-DTAC $\left(X_{\text {DTAC }}=0.6\right)$ & 144 & 0.13 \\
\hline HED-SDS $X_{\mathrm{SDS}}=0$ & $4.27^{b)}$ & $0.37^{c)}$ \\
\hline 0.2 & 16.8 & 0.37 \\
\hline 0.4 & 30.2 & 0.37 \\
\hline 0.6 & 47.0 & 0.37 \\
\hline 0.8 & 68.2 & 0.37 \\
\hline 1.0 & 118 & 0.34 \\
\hline HED-DTAC $X_{\text {DTAC }}=0.2$ & $46.0^{b)}$ & $0.13^{c)}$ \\
\hline 0.4 & 76.6 & 0.13 \\
\hline 0.6 & 142 & 0.13 \\
\hline 0.8 & 180 & 0.13 \\
\hline 1.0 & 948 & 0.14 \\
\hline
\end{tabular}

a) Obtained graphically. b) Obtained by calculation using Eq. 3. c) Presumed value.

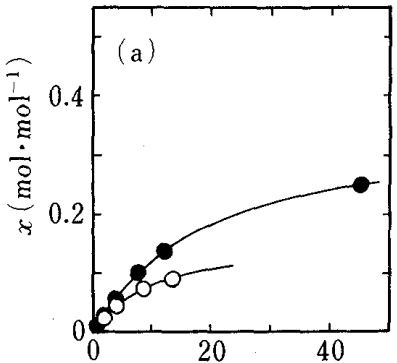

$\left[D_{\mathrm{f}}\right]\left(\mathrm{mmol} \cdot \mathrm{dm}^{-3}\right)$

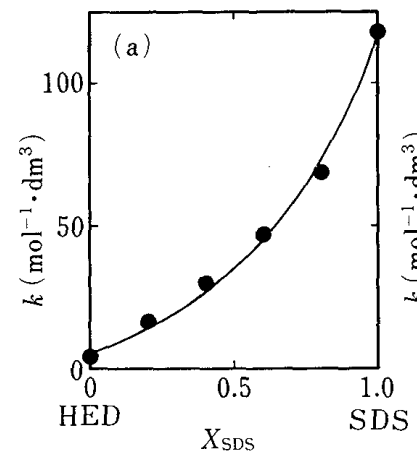

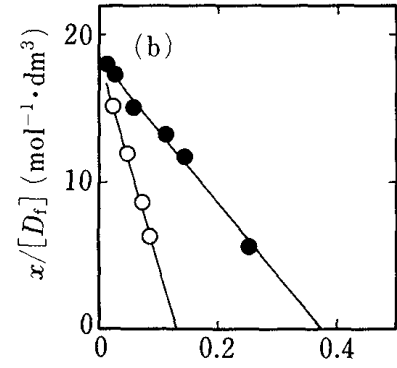

$x\left(\mathrm{~mol} \cdot \mathrm{mol}^{-1}\right)$

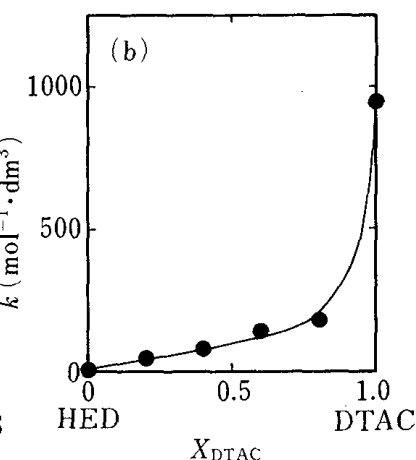

Fig. 8. Relation between $x$ and $\left[D_{\mathrm{t}}\right](\mathrm{a})$, and the Scatchard Plot (b) for the Interaction of Ach with Mixed Surfactant Systems

Mixed surfactant system: $O, X_{\text {DTAC }}=0.6$; $X_{\mathrm{SDS}}=0.6$.

Total surfactant concentration: $20 \mathrm{mmol} \cdot \mathrm{dm}^{-3}$.

Fig. 9. Binding Constant, $k$, for HED-SDS Surfactant Systems (a) and HED-DTAC Surfactant Systems (b)

Total surfactant concentration: $20 \mathrm{mmol} \cdot \mathrm{dm}^{-3}$

The solid line shown in (a) is the theoretical curve given by the Gouy-Chapman equation for $\sigma=$ $-2.150 \times 10^{-6} \cdot X_{\mathrm{SDS}}$ and by Eq 5 .

micelles calculated from the values of Fig. 5 are shown in Fig. 8. The values of $k$ and $x_{\infty}$ for the SDS-HED system $\left(X_{\text {SDS }}=0.6\right)$ and the DTAC-HED system $\left(X_{\text {DTAC }}=0.6\right)$ were obtained (footnote $a$ in Table I).

The $k$ for the HED-SDS system can be calculated from the $\left[D_{\mathrm{f}}\right]$ and $\left[D_{\mathrm{b}}\right]$ data shown in Fig. 4(a) by using Eq. 3 derived from Eq. 1,

$$
k_{(0 \text { or } x)}=\frac{1}{\left[D_{\mathrm{f}}\right]} \cdot \frac{\left[D_{\mathrm{b}}\right]}{[S] x_{\infty}-\left[D_{\mathrm{b}}\right]}
$$

where $k_{x}$ is the $k$ of HED-SDS mixed micelles ( $x$ is the mole fraction of SDS, $0 \leqq x \leqq 1$ ), $k_{0}$ is the $k$ at $x=0$ in the absence of SDS, and the values of $x_{\infty}$ used are $0.34 \mathrm{~mol} \cdot \mathrm{mol}^{-1}$ for SDS 
micelles and $0.37 \mathrm{~mol} \cdot \mathrm{mol}^{-1}$ for HED and HED-SDS mixed micelles, which are the values obtained from the Scatchard plots shown in Figs. 6(b) and 8(b) for SDS micelles and HEDSDS mixed micelles $\left(X_{\mathrm{SDS}}=0.6\right)$. Furthermore, the values of $[S]$ used are $20 \mathrm{mmol} \cdot \mathrm{cm}^{-3}$ for HED and HED-SDS mixed micelles and $16 \mathrm{mmol} \cdot \mathrm{dm}^{-311 c)}$ for SDS micelles. The values of $k_{0.6}$ for HED-SDS mixed micelles $\left(X_{\mathrm{SDS}}=0.6\right)$ and $k_{1}$ for SDS micelles thus obtained were 47 and $118 \mathrm{~mol}^{-1} \cdot \mathrm{dm}^{3}$, respectively. These values are consistent with the values of 48 and $120 \mathrm{~mol}^{-1} \cdot \mathrm{dm}^{3}$ obtained graphically from the Scatchard plots shown in Figs. 8(b) and 6(b). The values calculated by using Eq. 3 are shown in Fig. 9, which includes the values for HEDDTAC mixed micelles obtained by a similar equation to Eq. 3.

\section{Discussion}

\section{$\boldsymbol{k}$ and $\boldsymbol{x}_{\infty}$ of Ach for the Micelles}

The values of $k$ and $x_{\infty}$ for the ionic micelles shown in Table I indicate that Ach has a weaker binding force but larger saturated adsorption amounts for SDS micelles than for DTAC micelles. This may be explained as follows: (1) for the SDS micelles, Ach molecules are bound at the surface of the micelles by the electrostatic attractive force between the positive charge of Ach and the negative charge of SDS, while (2) for the DTAC micelles, Ach molecules penetrate more deeply into the micelles. This is because DTAC micelles are more hydrophobic than SDS micelles, ${ }^{14)}$ leading to stronger hydrophobic binding between the hydrocarbon chains of Ach and DTAC. (In this case, it may be considered that the acetyl group of Ach also enters the palisade layer of the DTAC micelles.) In addition, the positive charge residing on the quaternary nitrogen atom of the cationic micelles is covered with methyl groups and is less exposed than the negative charge of anionic micelles. Thus, the large value of $k$ and small value of $x_{\infty}$ for the DTAC micelles can be interpreted satisfactorily.

Next, the value of $k$ for the negatively charged mixed micelle system is smaller than that for the micelles of SDS only. This is considered to be because the negative charge density at the surface of the SDS micelles is decreased by the admixture of HED, leading to a reduction of the electrostatic attractive force between Ach and the micelles. Regarding the surface potential and the surface charge density of the mixed micelle system composed of SDS and HED; we have already reported ${ }^{11 a}$ ) that the relation between the surface potential and the surface charge density can be expressed by the Gouy-Chapman theory and that the surface charge density is proportional to $X_{\text {SDS. }}$. The value of $x_{\infty}$ in the SDS-HED system is approximately the same as that in the system with SDS alone.

The value of $k$ in the DTAC-HED mixed micelle system is considerably smaller than that in the system of DTAC alone. This is considered to be because the hydrophobic interaction between Ach and the micelles is weakened because of the admixture of HED which has weaker hydrophobic character than DTAC. Furthermore, it is evident that the relation between $k$ and micelle composition cannot be explained from the viewpoint of electrostatic interaction because $k$ increases with mole fraction of DTAC. The value of $x_{\infty}$ in the DTACHED system is approximately the same as that in the system of DTAC alone.

\section{Relations among Binding Constant, Micellar Surface Potential and Micelle Composition for the HED-SDS System}

The binding constant of Ach to the HED-SDS mixed micelles, $k$, is considered to depend on the surface potential of the micelles, $\Delta \psi$. The relation is, therefore, written as follows:

$$
\frac{k_{x}}{k_{0}}=\exp \left(-z \boldsymbol{F}_{\mathrm{A}} \Delta \psi / \boldsymbol{R} T\right)
$$

where $z$ is the valency of $\operatorname{SDS}, \boldsymbol{F}_{\mathrm{A}}$ is the Faraday constant and $\boldsymbol{R}$ is the gas constant. By 

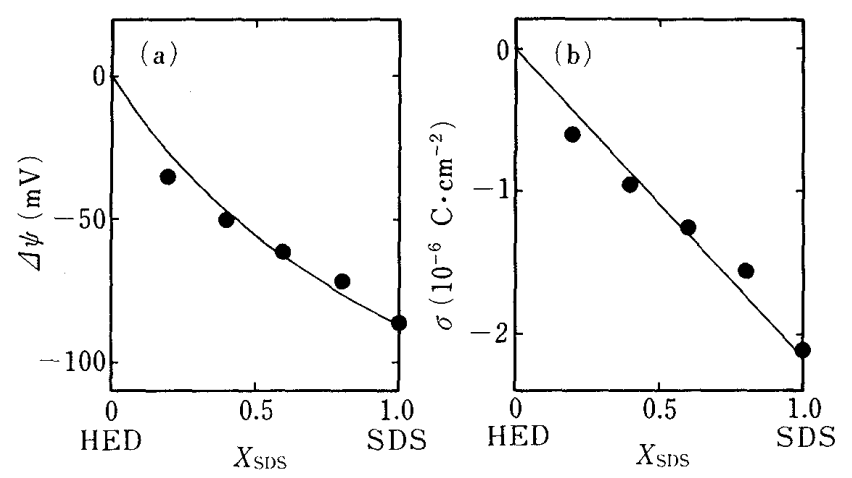

Fig. 10. Surface Potential, $\Delta \psi$, (a) and Surface Charge Density, $\sigma$, (b) for HED-SDS Surfactant Systems

Solid lines are the theoretical curves given by the Gouy-Chapman equation for $\sigma=-2.150 \times 10^{-6}$. $X_{\text {SDS. }}$

substituting $z=-1, F_{\mathrm{A}}=e \cdot N_{\mathrm{A}}=9.648 \times 10^{4}\left(\mathrm{C} \cdot \mathrm{mol}^{-1}\right), \quad R=k_{\mathrm{B}} N_{\mathrm{A}}=8.314\left(\mathrm{~J} \mathrm{~mol}^{-1} \cdot \mathrm{K}^{-1}\right)$, and $T=298.15 \mathrm{~K}$ at $25^{\circ} \mathrm{C}$,

$$
\Delta \psi=-59.16 \cdot \log \frac{k_{x}}{k_{0}}
$$

where $k_{x}$ or $k_{0}$ is expressed by Eq. 3 . The surface potential of the micelles, $\Delta \psi$, calculated by using Eq. 5, is plotted against the mole fraction of SDS in Fig. 10(a). The obtained value of $\Delta \psi$ for SDS micelles is $-85.2 \mathrm{mV}$ at ionic strength $5 \mathrm{mmol} \cdot \mathrm{dm}^{-3}$, and this is consistent with reported values of $-73 \mathrm{mV}$ at ionic strength $20 \mathrm{mmol} \cdot \mathrm{dm}^{-311 a)}$ and $-100.3 \mathrm{mV}$ at ionic strength $2.5 \mathrm{mmol} \cdot \mathrm{dm}^{-3} \cdot{ }^{11 b)}$

According to the Gouy-Chapman theory, the relation between the surface potential, $\Delta \psi$, and the surface charge density, $\sigma$, is expressed by Eq. 6 ,

$$
\frac{\sigma}{\mathrm{C} \cdot \mathrm{cm}^{-2}}=-5.8673 \times 10^{-6} \sqrt{\sum_{i}\left[\left(\frac{\mathrm{C}_{i}}{\mathrm{~mol} \cdot \mathrm{dm}^{-3}}\right)\left\{\exp \left(-0.03892 z_{i} \frac{\Delta \psi}{\mathrm{mV}}\right)-1\right\}\right]}
$$

where $78.54^{15)}$ is used as the value of relative dielectric constant of water at $25^{\circ} \mathrm{C}$. The relation between the surface charge density and the mole fraction of SDS is shown in Fig. 10(b). The value of $\sigma$ thus obtained was found to be proportional to $X_{\mathrm{SDS}}$, and the relation can be written as follows:

$$
\sigma=-2.150 \times 10^{-6} \cdot X_{\mathrm{SDS}}
$$

By using this relation, the theoretical values of the surface potential of the micelles and the binding constant were calculated by using the Gouy-Chapman Eq. 6 and Eq. 5, respectively, and the results are shown in Figs. 9(a) and 10(a) by solid lines, respectively. Thus, the relations among binding constant, micellar surface potential and micelle composition were found to be represented well by the Gouy-Chapman theory. The increase of binding constant with mole fraction of SDS is, therefore, due to the increase of the negative potential $(-\Delta \psi)$.

The relations among the surface charge density, $|\sigma|$, the area occupied by one molecule in an SDS micelle and the dissociation degree, $\alpha$, of SDS micelles can be expressed by Eq. 8 :

$$
\alpha=\frac{|\sigma| \cdot \mathrm{A}}{e}
$$

where $e=1.6021 \times 10^{-19} \mathrm{C}$. The degree of dissociation, $\alpha$, of SDS micelles calculated from the value of the area occupied by one SDS molecule, $58 \AA^{2},{ }^{16)}$ as described previously ${ }^{11 a)}$ is approximately $8 \%$. This value $(\alpha=8 \%)$ is nearly equal to the value $(\alpha=10 \%)$ obtained in our previous paper, ${ }^{11 a)}$ so that the results obtained in this paper seem to be valid.

In the DTAC-HED mixed micelle system, the binding constant, $k$, cannot be explained in terms of the electrostatic interaction as mentioned previously, so that calculation as done for the SDS-HED mixed system was not carried out. 


\section{Distribution Coefficient of Ach for Micelles}

The distribution coefficient for Ach between micellar and aqueous phases, $K$, is given by

$$
K=\frac{D_{\mathrm{b}} / V_{\mathrm{m}}}{D_{\mathrm{f}} / V_{\mathrm{a}}}
$$

where $D_{\mathrm{b}}$ is the amount of Ach in the micellar phase, $D_{\mathrm{f}}$ is the amount of Ach in the aqueous phase, $V_{\mathrm{m}}$ is the volume of the micellar phase and $V_{\mathrm{a}}$ is the volume of the aqueous phase. The value of $D_{\mathrm{f}} / V_{\mathrm{a}}$ means the concentration of Ach in the aqueous phase and can be determined directly by analyzing the filtrate obtained by ultrafiltration. The value of $V_{\mathrm{m}}$ is generally not known but is considered to be proportional to the amount of surfactant, $S$, over a limited concentration range, and thus, using $k^{\prime}$ as the proportional constant,

$$
K=\frac{D_{\mathrm{b}} / k^{\prime} \cdot S}{D_{\mathrm{f}} / V_{\mathrm{a}}}
$$

or

$$
K^{\prime}=K \cdot k^{\prime}=\frac{\left[D_{\mathbf{b}}\right] /[S]}{\left[D_{\mathrm{f}}\right]}
$$

where $K^{\prime}$ is the quantity usually calculated as the apparent distribution coefficient. Here, $\left[D_{\mathrm{b}}\right]=D_{\mathrm{b}} / V_{\mathrm{a}},\left[D_{\mathrm{f}}\right]=D_{\mathrm{f}} / V_{\mathrm{a}}$, and $[S]$ is the concentration of micellar surfactant, which can be obtained accurately from the values of $\mathrm{cmc}$ of the surfactant in the presence of various concentrations of Ach. ${ }^{11 c)}$ Furthermore, the relation between Eq. 11 and the Langmuir adsorption equation can be written as follows:

$$
K^{\prime}=k\left(x_{\infty}-x\right)
$$

or Eq. 12 can be approximated as Eq. 13, because $x_{\infty} \gg x$ under our experimental condition $\left(\left[D_{\mathrm{b}}\right]+\left[D_{\mathrm{f}}\right]=5 \mathrm{mmol} \cdot \mathrm{dm}^{-3}, x=\left[D_{\mathrm{b}}\right] /[S]\right)$,

$$
K^{\prime}=k \cdot x_{\infty}
$$

The apparent distribution coefficient of Ach for micelles is, therefore, calculated from the values of $k$ and $x_{\infty}$ obtained in the previous section, and it is evident that $K^{\prime}$ is independent of $[S]$. The values of $K^{\prime}$ calculated by using Eq. 13 for HED-SDS mixed micelles and for HEDDTAC mixed micelles are given in Table II (footnote $a$ ), which also lists the values of $K^{\prime}$ (for SDS and DTAC micelles) calculated by using Eq. 11 from the data shown in Fig. 3 (footnote $b$ ). The values of $K^{\prime}$ thus obtained for sufficient concentrations of SDS and DTAC were found to be independent of the concentration of surfactant at a total concentration of Ach of $5 \mathrm{mmol} \cdot \mathrm{dm}^{-3}$. These values obtained by using Eq. 11 (footnote $b$ in Table II) are consistent

TABLE II. Apparent Distribution Coefficient, $K^{\prime}$, of Ach for Micelles

\begin{tabular}{ccccc}
\hline \hline Surfactant micelles & $K^{\prime}\left(\mathrm{mol}^{-1} \cdot \mathrm{dm}^{3}\right)$ & Surfactant micelles & $K^{\prime}\left(\mathrm{mol}^{-1} \cdot \mathrm{dm}^{3}\right)$ \\
\hline HED-SDS & & HED-DTAC & \\
$X_{\text {SDS }}=0$ & $1.6^{a)}$ & $X_{\text {DTAC }}=0.2$ & $6.0^{a)}$ \\
0.2 & 6.2 & 0.4 & 10 & 18 \\
0.4 & 11 & 0.6 & 23 & $130^{b)}$ \\
0.6 & 17 & 1.0 & 132 & \\
0.8 & 25 & & & \\
1.0 & 40 & $40^{b)}$ & & \\
\hline
\end{tabular}

a) Calculated by Eq. 13. b) Calculated by Eq. 11 from the data shown in Fig. 3 . 
with the values obtained by using Eq. 13 (footnote $a$ in Table II). Therefore, it was found that the apparent distribution coefficient can be expressed by means of the Langmuir adsorption equation.

\section{Conclusion}

The distribution of Ach between micellar and aqueous phases has been measured and it has been found that Ach is distributed not only in SDS micelles but also in DTAC micelles. Furthermore, Ach has a stronger binding force for DTAC micelles than for SDS micelles, although the saturated adsorption amount is lower. This indicates that Ach molecules penetrate more deeply in the DTAC micelles and bind tightly by hydrophobic binding. This is considered to be because cationic micelles are more hydrophobic ${ }^{14)}$ than anionic micelles which have similar molecular weight. In the systems of Ach and HED-SDS mixed micelles, the binding constant increased with the mole fraction of SDS. This is considered to be due to the increase of the negative surface potential $(-\Delta \psi)$ of the micelles, which increases the electrostatic attraction between the positive charge of Ach and the negative charge of the micelles. The relation between the binding constant of Ach to the HED-SDS mixed micelles and the surface potential of the micelles was well described by the Gouy-Chapman theory.

\section{References and Notes}

1) Presented at the 34th Annual Meeting of the Kinki Chapter, Pharmaceutical Society of Japan, Nishinomiya, November 1984

2) J. Gettins, D. Hall, P. L. Jobling, J. E. Rassing and E. Wyn-Jones, J. Chem. Soc., Faraday Trans. 2, 74, 1957 (1978).

3) E. B. Abuin and E. A. Lissi, J. Colloid Interface Sci., 95, 198 (1983).

4) R. D. Lisi, C. Genova and V. T. Liveri, J. Colloid Interface Sci., 95, 428 (1983).

5) K. Hayase and S. Hayano, Bull. Chem. Soc. Jpn., 50, 83 (1977).

6) a) S. J. A. Kazmi and A. G. Mitchell, J. Pharm. Sci., 60, 1422 (1971); b) H. Kurz, H. Trunk and B. Weitz, Arzneim. Forsch., 27, 1373 (1977).

7) I. W. Osborne-Lee, R. S. Schechter and W. H. Wade, J. Colloid Interface Sci., 94, 179 (1983).

8) S. J. Dougherty and J. C. Berg, J. Colloid Interface Sci., 48, 110 (1974).

9) a) T. Shimamoto, Y. Ogawa and N. Ohkura, Chem. Pharm. Bull., 21, $316(1973)$; b) T. Shimamoto and Y. Ogawa, ibid., 23, 3088 (1975); c) T. Shimamoto, H. Mima and M. Nakagaki, ibid., 27, 1995 (1979); d) Idem, ibid., 27, 2557 (1979); e) T. Shimamoto and H. Mima, ibid., 27, 2602 (1979).

10) M. Nakagaki and S. Yokoyama, Bull. Chem. Soc. Jpn., in preparation.

11) a) M. Nakagaki, S. Yokoyama and I. Yamamoto, Nippon Kagaku Kaishi, 1982, 1865; b) M. Nakagaki and S. Yokoyama, J. Pharm. Sci., accepted; c) M. Nakagaki and S. Yokoyama, Bull. Chem. Soc. Jpn., 58, 753 (1985).

12) W. F. Blatt, S. M. Robinson and H. J. Bixler, Anal. Biochem., 26, 151 (1968).

13) S. Hestrin, J. Biol. Chem., 180, 249 (1949).

14) J. H. Fendler and E. J. Fendler, "Catalysis in Micellar and Macromolecular Systems," Academic Press, New York, 1975.

15) Kagaku Binran, ed. by Nippon Kagaku Kai, Maruzen, Japan, 1966, p. 1005.

16) H. V. Tartar, J. Phys. Chem., 59, 1195 (1955). 